\title{
Epigenetic Regulation of Hepatitis B Virus Covalently Closed Circular DNA: Implications for Epigenetic Therapy against Chronic Hepatitis B
}

\author{
Xupeng Hong ${ }^{1 * \infty}$, Elena S. Kim ${ }^{2}$, Haitao Guo ${ }^{2 *}$
}

1. Department of Microbiology and Immunology, Georgetown University Medical Center, Washington, DC 20057, USA

2. Department of Microbiology and Immunology, Indiana University School of Medicine, Indianapolis, IN 46202, USA

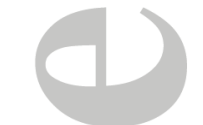

a Current Address: Department of Microbiology and Immunology, Penn State University College of Medicine, Hershey, PA 17033, USA

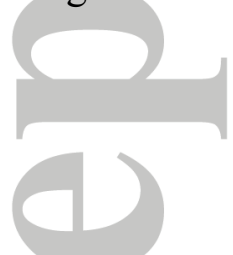

Key words: HBV cccDNA, DNA methylation, histone modification, epigenetic therapy

*Corresponding author: Haitao Guo, Email: haitguo@iupui.edu; Xupeng Hong, Email: xh81@georgetown.edu

This is the author's manuscript of the article published in final edited form as:

Hong, X., Kim, E. S. and Guo, H. (), Epigenetic Regulation of Hepatitis B Virus Covalently Closed Circular DNA: Implications for Epigenetic Therapy against Chronic Hepatitis B. Hepatology. Accepted Author Manuscript. http://dx.doi.org/10.1002/hep.29479 


\section{List of Abbreviations:}

HBV, hepatitis B virus; CHB, chronic hepatitis B; cccDNA, covalently closed circular DNA; pgRNA, pregenomic RNA; pol, polymerase; HBc, HBV core protein; HBsAg, HBV surface antigen; HCC, hepatocellular carcinoma; PTM, post-translational modification; H3, histone 3; H4, histone 4; DNMT, DNA methyltransferase, HDAC, histone deacetylase; IFN, interferon; NUC, nucleos(t)ide analogue; DNMTi, DNA methyltransferase inhibitor; HDACi, histone deacetylase inhibitor; CTL, cytotoxic T cell

\section{Financial Support}

This study is supported by NIH grant R01AI094474, R01AI110762, and R01AI123271.

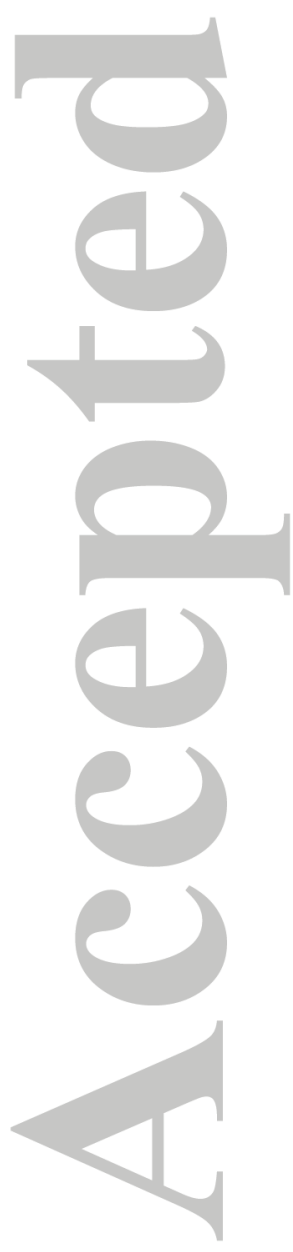




\section{ABSTRACT}

Hepatitis B virus (HBV) infection represents a significant public health burden worldwide. Although current therapeutics manage to control the disease progression, lifelong treatment and surveillance are required because drug resistance develops during treatment and reactivations frequently occur following medication cessation. Thus, the occurrence of hepatocellular carcinoma $(\mathrm{HCC})$ is decreased but not eliminated. One major reason for the treatment failure is the inability to eradicate or inactivate the viral covalently closed circular DNA (cccDNA) which is a stable episomal form of viral genome decorated with host histones and non-histone proteins. Accumulating evidence suggests that epigenetic modifications of cccDNA contribute to viral replication and the outcome of chronic HBV infection. Here, we summarize the progress on HBV epigenetics research and the therapeutic implications for chronic HBV infection by learning from the epigenetic therapies for cancer and other viral diseases, which may open a new venue to cure the chronic hepatitis B. 


\section{INTRODUCTION}

Hepatitis B virus (HBV) infection remains a significant public health problem worldwide. The outcome of HBV infection is determined by multiple host and virus factors and the infection can be acute, chronic or occult(1). Chronic hepatitis B (CHB) affects more than 240 million individuals worldwide which are at risk of developing fibrosis, cirrhosis, liver failure and hepatocellular carcinoma (HCC)(2). The therapeutic goal of CHB is to decrease the risk of liver diseases progression and prevent its detrimental clinical sequelae, which can be partly achieved by sustained suppression of the virus replication, or ideally by eradicating the virus infection(3). Currently, US FDA-approved drugs for CHB treatment include two formulations of interferon alpha (standard and pegylated, IFN- $\alpha$ and $\operatorname{pegIFN}-\alpha$, respectively) that modulate the host antiviral immune response, and five nucleos(t)ide analogues (NUCs) including lamivudine, adefovir, entecavir, telbivudine and tenofovir that inhibit the HBV reverse transcription(4). Current therapies could lead to suppression of viral replication, resulting in normalization of serum alanine transaminase and improvement of liver histology(5). However, even prolonged treatments rarely cure the chronic infection, drug resistance occurs during treatment of NUCs, and virological relapse is common after treatment cessation(4). Strong evidence suggests that the lack of curativeness of current antiviral therapies is due to the inability to eliminate the episomal HBV covalently closed circular DNA (cccDNA), which serves as the persistent form of the viral genome and the transcription template for virus reproduction(6,7).

The term 'epigenetics' refers specifically to the heritable alterations in gene expression that occurs to chromosome without changes in DNA sequence(8). Epigenetic regulation mechanisms include but may not be limited to DNA methylation, histone modifications, chromatin 
remodeling and noncoding RNA (ncRNA) interference. HBV cccDNA exists in the nucleus as multiple copies of nucleosome-decorated minichromosomes $(9,10)$, which implies that epigenetic modifications may influence HBV replication and persistent infection. Epigenetic therapy, a novel strategy targeting epigenetic modifications, was first applied successfully to leukemia treatment(11). To date, evidence is accumulating that the epigenetic mechanisms are responsible for $\mathrm{HBV}$ persistence and clearance, which indicates that epigenetic therapy possesses potentials to be a novel approach to treat $\mathrm{CHB}$ and may even cure it. In this review, we attempt to provide an overview of the epigenetic regulations of $\mathrm{HBV}$ infection and the possible therapeutic strategies for $\mathrm{CHB}$ from the lessons of cancer and other viral diseases.

\section{HBV LIFE CYCLE AND CCCDNA BIOLOGY}

HBV is the prototype of the Hepadnaviridae family, a small enveloped DNA virus with a partially double-stranded relaxed circular (rc) genome of $3.2 \mathrm{~kb}$. The infection begins when HBV virion binds with low affinity to heparansulfate proteoglycans (HSPG), followed by specific binding of preS1 domain of the large surface antigen (LHBsAg) with the viral receptor on the surface of the hepatocyte, which is the sodium taurocholate cotransporting polypeptide (NTCP)(12). However, how the virus is endocytosed into the cytoplasm has not been completely demonstrated(13). After uncoating, rcDNA in the HBV nucleocapsid is translocated into the nucleus and converted into cccDNA minichromosome. The detailed molecular mechanisms of nucleocapsid disassembly and cccDNA biosynthesis remain elusive. Using cccDNA as transcription template, HBV transcripts are generated by host RNA polymerase II machinery, including the $3.5 \mathrm{~kb}$ pregenomic RNA (pgRNA) which encodes viral polymerase (pol) and HBV core protein $(\mathrm{HBc})$, the $3.5 \mathrm{~kb}$ preCore mRNA for translation of e-antigen ( $\mathrm{HBeAg})$, the 
2.4/2.1 kb preS/S mRNA encoding the large (L), middle (M) and small (S) HBsAg, and a 0.7kb mRNA encoding the regulatory protein $\operatorname{HBx}(14)$. In the cytoplasm, $\mathrm{HBc}$ proteins package pol and pgRNA and assemble into nucleocapsid, in which the pgRNA is reverse transcribed into rcDNA by the pol. After rcDNA is made, the capsid can be either coated by viral envelope for virion egress or redirected to the nucleus to replenish the cccDNA pool (Fig.1).

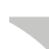

In the nucleus, similar to other nuclear viral episomal DNA, HBV cccDNA exhibits as individual minichromosomes with cellular chromatin-like structures $(9,10)$. Nucleosomes are nonrandomly positioned on the cccDNA of duck hepatitis B virus (DHBV), suggesting that, like host cellular chromatin, nucleopositioning and histone modifications of the cccDNA may regulate its transcription(15). cccDNA minichromosome is stably maintained in quiescent hepatocytes with a long half-life and is responsible for the persistence of infection during the natural course of chronic infection and prolonged antiviral treatment $(7,16)$. HBV is not a cytopathic virus per se and most of hepatocytes in the adult liver are quiescent, however, the proliferation rate of hepatocytes can be greatly enhanced by liver inflammation and regeneration caused by viral infection $(17,18)$. cccDNA appears not to be tethered to the host chromosome during mitosis, thus cccDNA are randomly partitioned into daughter cells and some of them are lost during cell division(19). However, a complete loss of cccDNA solely by hepatocyte proliferation cannot be achieved in an immunodeficient mouse model without blocking virus spread(19,20). It is now accepted that, during the recovery phase of an acute HBV infection, both $\mathrm{CD}^{+}$cytotoxic $\mathrm{T}$ cell (CTL)-mediated cytolytic and noncytolytic antiviral responses are responsible for the clearance of infection(14,21). Interestingly, cccDNA still can be detected in a significant percentage of fully recovered ("cleared") patients $(7,22)$, and reactivation of HBV infection in these patients 
under immunosuppression conditions or chemotherapy is not uncommon(23), suggesting that cccDNA is inherently stable and it might be difficult to get rid of the very last copy, but silencing the activity of cccDNA, such as epigenetic modifications, might be a more realistic and practical way to control hepatitis B.

\section{EPIGENETIC CONTROL OF HBV INFECTION}

\section{HBV DNA Methylation}

DNA methylation usually occurs at the 5' position of cytosine ring within $\mathrm{CpG}$ dinucleotides by DNA methyltransferases (DNMTs) in mammalian cells and is generally associated with transcription silencing(24).

The HBV cccDNA genome contains three predicted CpG islands: (1) the region overlapping the start site of the $S$ gene (Island 1); (2) the region encompassing enhancer I, the $H B x$ gene promoter $(\mathrm{Xp})$, and the core promoter (Cp) (Island 2); (3) the region harboring the Sp1 promoter and the start codon of the pol gene (Island 3) (Fig.2)(25,26). Among the 10 distinct genotypes (A-J) of $\mathrm{HBV}$, the methylation rates in Island 1 are variable, while Island 2 and 3 are more conserved across genotypes(26). Methylation of the $\mathrm{CpG}$ islands on $\mathrm{HBV}$ cccDNA regulates HBV replication and gene expression. Zhang et al., demonstrated that cccDNA CpG Island 1 is seldom methylated, methylation of CpG Island 2 is associated with low viremia, and methylation of CpG Island 3 may contribute to a lower serum HBsAg level in CHB patients(27). Moreover, the methylation of $\mathrm{CpG}$ Island 3 is significantly correlated with hepatocarcinogenesis(28). In 
vitro study has shown that $\mathrm{CpG}$ Island 2 methylations negatively affected pgRNA transcription and reduced viral DNA replication(27).

The role of DNMTs in HBV DNA methylation has not been clearly identified. In vitro studies reported that DNMT1, DNMT2, and DNMT3 expression is upregulated in response to HBV, leading to viral and host DNA hypermethylation and decreased HBV replication(29), but the underlying mechanism for HBV-mediated upregulation of DNMTs is unclear. It is worth noting that DNA methylation only occurs on the nuclear HBV DNA forms, but not the histone-free cytoplasmic core DNA or virion $\operatorname{DNA}(25,27,28)$. In the nucleus, the integration of HBV DNA into the human chromosome was frequently detected in HCCs from CHB patients, although it's not essential of the viral life cycle(30). The integrated HBV DNA is replication-defective, but it can express HBsAg and/or an HBx fusion protein, which may contribute to virus persistence and hepatocarcinogenesis(30). A previous study has demonstrated that the integrated HBV DNA are methylated and silenced in the SNU398 HCC cell line(28). However, whether the episomal cccDNA and the integrated DNA are methylated by same or different mechanism remains unknown. Viral DNA methylation has been identified as a host defense mechanism since it often leads to the downregulation of viral gene expression(31), thus methylating the nuclear HBV DNA may represent a novel antiviral strategy for HBV treatment.

\section{Histone modifications of the cccDNA Minichromosome}

Nucleosome-associated histones can undergo a great deal of post-translational modifications (PTMs), which include acetylation, methylation, phosphorylation, ubiquitination, and many others within their N-terminal tails(32). The enzymes that add and remove such modifications 
are histone acetyltransferases (HATs) and deacetylases (HDACs), lysine (K) methyltransferases (KMTs) and demethylases (KDMs), kinases and phosphatases, ubiquitin ligases and deubiquitinases, and so on(32,33). Genome-wide studies revealed that various combinations of modifications in a specific genomic region can lead to either activation or repression of gene expression (32). Histone acetylation and methylation have been well characterized. For instance, trimethylation of lysine 4, 36 or 79 on $\mathrm{H} 3$ (H3K4me3, H3K36me3, and H3K79me3, respectively), and acetylation of $\mathrm{H} 3 \mathrm{~K} 9, \mathrm{H} 3 \mathrm{~K} 27$ and $\mathrm{H} 3 \mathrm{~K} 122$ (H3K9ac, H3K27ac and H3K122ac) contribute to gene activation, whereas di- or trimethylation of H3K9 (H3K9me2 and H3K9me3) and trimethylation of $\mathrm{H} 3 \mathrm{~K} 27$ (H3K27me3) lead to transcriptional repression(32-34). Similarly, arginine methylation also plays a role in gene expression. Asymmetrical dimethylation on H4R3 (H4R3me2a) is associated with transcription activation, while symmetrical methylation on H4R3 (H4R3me2s) is linked to transcriptional repression(35).

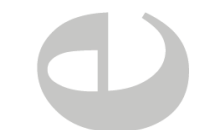

The PTMs of histones and non-histone proteins that either directly bind to cccDNA sequence or bind to cccDNA minichromosome through protein-protein interactions have become a hot area of research. A growing body of evidence suggests that histone modifications on cccDNA regulate the viral transcription. The $\mathrm{HBc}$ and $\mathrm{HBx}$ proteins, $\mathrm{H} 3$ and $\mathrm{H} 4$ histones, cellular transcription factors (CREB, ATF, YY1, STAT1 and STAT2), and chromatin modification enzymes (PCAF/GCN5, p300/CBP, HDAC1, SIRT1, PRMT1, PRMT5 and EZH2) have been shown to be associated with cccDNA by using chromatin immunoprecipitation (ChIP) assays (10,36-40) (Fig.3). Hypoacetylation of the cccDNA-associated H3 and H4 histones and the recruitment of the cellular HDAC1 onto cccDNA are associated with low HBV replication in vitro and in vivo(37). The presence of class I/II histone deacetylase inhibitors (HDACis), 
valproic acid and trichostatin A (TSA), have been shown to induce an evident increase of cccDNA-bound acetylated $\mathrm{H} 3$ and $\mathrm{H} 4$ histones and $\mathrm{HBV}$ replication in the linear monomeric HBV DNA transfected Huh7 cells(37), suggesting a direct effect of the PTMs on HBV transcription. However, another study using DHBV stable cell line system showed that certain HDACis, including TSA, suppressed cccDNA transcription and reduced DHBV replication, indicating that a cellular function sensitive to HDACi might be required for DHBV cccDNA transcription(41). Such discrepancy between these two studies might be due to virus-specific (HBV vs. DHBV) and/or cell-specific (human vs. avian) reasons. Recently, the first genomewide map of PTMs on cccDNA chromatin revealed high levels of PTMs associated with active transcription (H3K4me3, H3K27ac, and H3K122ac) enriched at specific sites within the HBV genome from infected cell cultures and liver tissue, but very low levels of PTMs linked to transcriptional repression even at silent HBV promoters (42), suggesting_that the transcription activity of cccDNA is predominantly determined by those transcriptionally active PTMs.

H3K4me3 modification by MLL3, a component of ASCOM complex, has been shown to be critical for activating NTCP gene transcription by nuclear receptors in HepG2 cells(43). Considering that NTCP is a functional receptor for HBV, H3K4me3 modification of NTCP promoter by MLL3 may facilitate HBV infection in vivo. In line with this, Tropberger and colleagues found that cccDNA possesses a high level of $\mathrm{H} 3 \mathrm{~K} 4 \mathrm{me} 3$ (42), which suggests that MLL3 might be involved in shaping the histone PTMs of cccDNA. H3K27ac blocks polycombmediated $\mathrm{H} 3 \mathrm{~K} 27 \mathrm{me} 3$, and $\mathrm{H} 3 \mathrm{~K} 27 \mathrm{ac}$ is also widely used to identify active enhancers(44). EZH2 is a subunit of PRC2 and has the KMT activity on H3K27. Interestingly, a recent knockdown study of EZH2 showed the upregulation of $\mathrm{HBeAg}$ and $\mathrm{HBsAg}$, indicating a repressive function 
of EZH2 on HBV gene expression(40). H3K122ac is enriched at genetic elements associated with transcriptionally active genomes in vivo and is catalyzed by the transcriptional coactivator p300/CBP(45). Despite the coexistence of H3K122ac with other H3 tail acetylation in the same nucleosome, H3K122ac alone can be sufficient to stimulate transcription(46). According to the RNAi-based screening of known histone methyltransferases (SUV39H, G9a, EZH2, and PRMT5) and demethylases (LSD1, KDM5A, and KDM2A), Zhang et al., revealed that the PRMT5mediated H4R3me2s repressed cccDNA transcription. In addition, PRMT5-mediated H4R3me2s interacted with $\mathrm{HBc}$ and the Brg1-based hSWI/SNF chromatin remodeler, which accounted for the reduced binding of RNA polymerase II to $\operatorname{cccDNA}(40)$.

The viral regulatory protein HBx serves as a promiscuous transactivator of the viral and cellular promoters. HBx activates the transcription of host genes by interacting directly with nuclear transcription factors or by activating various signal transduction pathways in the cytoplasm and has been proven to be a potent epigenetic modifying factor in liver(47). HBx has been shown to activate $\mathrm{HBV}$ transcription through its recruitment onto cccDNA by host PCAF/GCN5, p300, and CBP acetyltransferases; and inhibit cellular factors involved in chromatin regulation, such as PP1/HDAC1 complex(38,48) (Fig.3A). Conversely, in the absence of HBx, the rapid hyperacetylation of cccDNA-bound histones by p300 is severely impaired, but the deacetylases HDAC1 and SIRT1 are recruited to cccDNA, leading to the suppression of cccDNA transcription and viral replication(38) (Fig. 3B). In addition, without HBx, the silenced HBV genome is not only associated with histone deacetylation but also with the deposition of repressive markers (H3K9me2 and $\mathrm{H} 3 \mathrm{~K} 9 \mathrm{me} 3)$ by SETDB1(48). Recently, HBx was shown to interact with DNA damage-binding protein 1 (DDB1) and recruit the cullin 4A-RING E3 
ubiquitin ligase (CRL4), the assembled CRL4-DDB1 complex ubiquitinates the Smc5/6 complex for proteasomal degradation to activate cccDNA transcription $(49,50)$, indicating that Smc5/6 complex may epigenetically silence cccDNA through histone PTMs in the absence of $\mathrm{HBx}(51)$ (Fig.3).

\section{(Fig.3).}

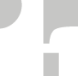

Although a series of histone modifications of HBV cccDNA minichromosome have been reported, the cccDNA-specific PTM enzymes have not been identified yet. We summarize herein the KMTs, KDMs, HATs, HDACs, and other factors determined in cancer research (Table 1), which may provide candidates for the future study of PTMs of HBV cccDNA-associated histones.

\section{DEVELOPMENT OF EPIGENETIC THERAPY FOR CHRONIC HBV INFECTION}

The pool of cccDNA is highly stable and persists in the hepatocytes. Currently available and experimental antivirals are interferons, inhibitors of reverse transcription, allosteric modulators of capsid assembly, cccDNA formation inhibitors, inhibitors of virus entry or release of viral or subviral particles, but their impact on the reduction of pre-existing cccDNA is limited(4,5). Alternatively, further understanding of epigenetic mechanisms in the HBV infection may promote the development of "epigenetic therapy" to hopefully silence HBV in infected hepatocytes. Drugs against epigenetic targets ("epidrugs") have been developed and some have been approved for selected cancers. Epigenetic discoveries continually define not only promising new targets for anti-cancer and antiviral therapy but also the ways to repurpose drugs already used in the clinic. The elucidation of these alteration pathways has attracted much attention to the dysregulation of epigenetics in disease and also the development of novel epidrugs. In principle, 
epigenetic therapy generally targets one or more of three protein categories: Writers, enzymes that establish epigenetic marks (e.g. DNMTs and HATs); Readers, proteins which recognize epigenetic regulations (e.g. proteins of the bromo and extra terminal family, BET, which are readers of histone acetylation) and may bring in other protein complexes to change gene expression; and Erasers, enzymes that remove epigenetic marks (e.g. HDACs and KDMs)(8) (Table 1). A growing body of studies on the epigenetic therapies in chronic viral infections, such as HIV and $\operatorname{EBV}(52,53)$, suggest that epigenetic therapy might be a promising therapeutic strategy for CHB.

\section{Targeting DNA methylation of cccDNA}

DNA methylation of viral genomes has been identified as a host defense mechanism(31). Increased expression of DNMTs has been reported to facilitate the methylation of the viral genome to impede HBV replication in vitro(29), which suggests that DNMT could be utilized to inhibit cccDNA transcription. However, on the other hand, host DNA methylation has been shown to be a major mechanism for inactivating relevant tumor suppressor genes in $\mathrm{HCC}(54)$, indicating an impossibility to directly use strong demethylation agents in the treatment of HCC. 5-azacytidine and 5-aza-2'-deoxycytidine are FDA-approved DNMT inhibitors (DNMTis). However, Azacitidine is contraindicated in patients with advanced hepatic tumors due to potential hepatotoxic effect(55). Therefore, for HBV-related HCC, the balance between blocking HBV infection and inhibition of host tumor-suppressor genes should be taken into careful consideration in the development of DNMT-based therapeutics. Several molecules interfering with the DNMTs activity are under the various phases of clinical trials for the treatment of several kinds of cancers(56), their effects on HBV cccDNA and HCC await further 
investigations. Studies with several cancer cell lines demonstrated that (-)-epigallocatechin-3gallate (EGCG) can alter DNMTs activity(57). In addition, EGCG also has been shown to inhibit HBV entry and induce anti-HBV autophagy response $(58,59)$, indicating that EGCG may be beneficial to HBV-related HCC patients.

It has been reported that HBV infection induced de novo methylation and decreased expression of antiviral cytokine IL-4(60). Furthermore, DNMTis have been shown to upregulate cytokines as well as JAK/STAT and IFN signaling pathways in cancer patients(61). Hence, DNMT-based epigenetic therapy may also evoke the innate immunity in both HBV-infected cells and immune cells to systematically enhance the suppression of HBV replication.

\section{Targeting histone modifications of cccDNA}

Recognition of the role of histone PTMs in cccDNA transcription provides several potential therapeutic targets to inhibit the cccDNA. Although the genome-wide mapping of PTMs on cccDNA minichromosome showed that the levels of transcriptional active PTMs, but not the repressive PTMs, determine the activity of cccDNA(42); but these PTMs also play similar roles in host chromosome transcription. Thus, the cccDNA-specific PTM modifiers, if any, will hold great promise for the development of therapeutic targets. In this regard, a recent study demonstrated that a multifunctional protein arginine methyltransferase PRMT5 preferentially induced H4R3me2s on cccDNA to repress viral transcription, indicating that certain PTM enzymes may be able to distinguish the host chromosome from HBV cccDNA for chromatin modifications(40)._Furthermore, Tropberger et al., found that HAT inhibitor C646 blocked histone acetyltransferases p300/CBP for H3K27ac and H3K122ac and inhibited HBV 
transcription without measurable cytotoxicity in $\mathrm{PHHs}(42)$, which provides a proof of concept that epigenetic compounds may represent a novel class of HBV antivirals.

Here, we discuss the current anti-HBV therapies that may affect cccDNA histone modification, as well as other successful cases of epigenetic therapy for other diseases. Since 2006, a number of HDACis have been approved by the FDA as anti-cancer drugs (vorinostat, romidepsin, belinostat, panobinostat, etc.). Nowadays, the study of HCC treatment using epidrugs is a cutting-edge area of research. Inhibition of deacetylation by HDACis has been shown to enhance HBV transcription and replication in vitro $(37,62)$. In addition, HDACis may also potentially reactivate latent DNA virus infection in patients receiving it as anticancer drugs(63). IFN- $\alpha$ is the only approved immunomodulator for CHB treatment(4). Recent studies suggested that its antiviral function involves epigenetic modification of cccDNA minichromosome. When treating the HBV-infected cells and chimeric uPA/SCID mice with IFN- $\alpha$, the transcription activity of cccDNA was inhibited through the hypoacetylation of cccDNA-associated histones and the recruitment of transcriptional repressor complex YY1 and EZH2, and HDAC1 and SIRT1 onto cccDNA(36). The reduction of $\mathrm{H} 3 \mathrm{~K} 27 \mathrm{ac}$ and $\mathrm{H} 3 \mathrm{~K} 122 \mathrm{ac}$, which are markers of active transcription, was observed on cccDNA under IFN- $\alpha$ treatment, suggested that IFN- $\alpha$ could activate PTM-modifying enzymes and lead to a transcriptionally less permissive cccDNA chromatin state(42). Another study also indicated that avian IFN- $\alpha$ treatment interferes with the structure and function of DHBV cccDNA minichromosome by reducing the acetylation of both $\mathrm{H} 3$ and $\mathrm{H} 4$ histones(41). 
It is known that IFN- $\alpha$ engages the IFN- $\alpha / \beta$ receptor complex to modulate the transcription of the IFN stimulated genes (ISGs) via JAK/STAT signaling pathway to evoke innate antiviral responses against viral infection(64). However, IFN- $\alpha$ monotherapy shows low response rate with severe adverse effects in CHB patients(65). Considering that both the host ISG expression and viral cccDNA transcription are regulated by epigenetic modifications, combining IFN- $\alpha$ with epidrugs might be able to rapidly achieve a more potent inhibition of cccDNA and reduce side effects. "Shock and Kill" is one of the most discussed and tested therapeutic strategies against HIV-1, which refers to the induction of latent provirus by various latency-reversing agents, followed by elimination of the infected cells by immune system or by cell lysis induced by viral cytopathic effect and prevention of new infection by combined antiretroviral therapy(52). DNMT, KMT, and HDAC inhibitors have been employed in activating latent reservoir of HIV-1(66). In terms of $\mathrm{HBV}$, high-dose IFN- $\alpha$ treatment has been reported to lead to cccDNA degradation through APOBEC3A-mediated cytidine deamination of one strand of cccDNA, by which induces mismatch mutations in cccDNA for Apurinic/Apyrimidinic-endonuclease-1-mediated degradation(67). Under IFN-elicited antiviral responses, it is assumed that HDACis treatment will loosen the structure of cccDNA minichromosome for transcription and thus increase the possibilities for APOBEC3A to edit cccDNA for degradation. With such potential synergy between IFN- $\alpha$ and epigenetic treatment, possible drawbacks should be avoided to move the approach forward. Firstly, IFN-induced APOBEC3s may facilitate the mutation of HBV DNA to assist the viral evolution, potentially resulting in immune escape and acceleration of HCC development(68). Secondly, HDACis are notorious for their toxic effects on platelet counts in vivo, in addition to the risk of reactivation of latent virus, such as HSV-1 and EBV(63), perhaps enhancing HBV as well if the enhancement overrides IFN's antiviral effect under combination. If 
these problems can be solved, it may be then possible to repurpose HDACis as potential therapeutic agents against cccDNA in CHB patients.

\section{POTENTIAL FOR DEVELOPMENT OF EPIGENETIC THERAPY COMBINED WITH IMMUNOTHERAPY}

Previous studies revealed that epigenetic cancer therapy can modulate the expressions of tumor suppressor genes, tumor-associated antigens (TAAs), and genes involved in immune response. The genes encoding TAAs are essential for $\mathrm{T}$ cell activation and tumor recognition by the immune system(69). Epigenetic regulation of host immune cells development and differentiation has also been well described(70). DNMTis can induce the expression of MHC-I molecules, which present epitopes to T cells and direct CTLs to lyse tumor cells(71). HDACis can affect the polarization of naïve $\mathrm{CD}^{+} \mathrm{T}$ cells toward $\mathrm{Th} 1$ and $\mathrm{Th} 2$ subsets(72). Hypomethylating agents increase the production of cytokines, including IL-2, TNF- $\alpha$, and IFN- $\gamma$, to kill tumor cells(73). For HBV, T cell-derived cytokines, TNF- $\alpha$ and IFN- $\gamma$, which induce APOBEC3A and APOBEC3B, may contribute to the cccDNA loss in a non-cytolytic fashion(74). Meanwhile, epidrugs also facilitate the killing of tumor cells through stimulating NK cells and CTLs(69). It is well acknowledged that CTL exhaustion/tolerance contributes to $\mathrm{CHB}$, thus the epigenetic therapy may possess a chance to restore $\mathrm{T}$ cell functions against $\mathrm{HBV}$, and future epigenetic therapy combined with immunotherapy (including the aforementioned IFN- $\alpha$ therapy) may hold promise for curing $\mathrm{CHB}$.

\section{CONCLUSION AND PERSPECTIVE}


Since the term "epigenetics" was coined more than 70 years ago, study on epigenetic modifications in normal and disease biology has grown to a major field of research $(8,34,63)$. Accumulating evidence supports that epigenetic regulation of cccDNA plays important roles in HBV reproduction. The desired end-point of HBV therapy is the elimination of cccDNA, or at least a complete inactivation of cccDNA, in the host, which cannot be achieved by the current antivirals. All these urge a need for developing epigenetic HBV therapies to target cccDNA. To this end, screening of available epidrugs or larger compound libraries in cccDNA reporter cell lines or HBV infection cell culture systems would be the first attempt. In addition, the cccDNAbound $\mathrm{HBc}$ and $\mathrm{HBx}$ are also attractive antiviral targets. $\mathrm{HBc}$ and $\mathrm{HBx}$ destabilizers or allosteric modulators may potentially alter the epigenetics of cccDNA and lead to transcriptional silencing or other antiviral effects. On the other hand, the genetic and epigenetic alterations of host chromosome caused by HBV infection are believed to play important roles in viral persistence and the development of HCC in CHB patients. Hence, epigenetic therapy may be a "two birds, one stone" approach to treat both $\mathrm{CHB}$ and HBV-related HCC. In cancer therapies, epidrugs has demonstrated their therapeutic values, together with many other beneficial effects on the host, such as innate and adaptive immune activations and reduced chemotherapy resistance $(61,69)$. In addition, some epidrugs have synergy with other epigenetic therapies, chemotherapies or immunotherapies(61). According to the available information about cccDNA biology and epigenetic therapy as we discussed in this review, it is envisioned that, in the near future, cccDNA-specific epigenetic mechanisms can be fully elucidated, and cccDNA-specific epidrugs can be developed to treat and even cure CHB.

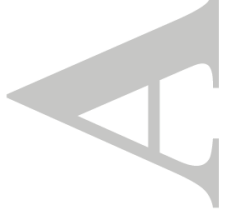




\section{REFERENCE}

1. Fattovich G, Bortolotti F, Donato F. Natural history of chronic hepatitis B: Special emphasis on disease progression and prognostic factors. J. Hepatol. 2008;48:335-352.

2. Schweitzer A, Horn J, Mikolajczyk RT, Krause G, Ott JJ. Estimations of worldwide prevalence of chronic hepatitis B virus infection: A systematic review of data published between 1965 and 2013. Lancet. 2015;386:1546-1555.

3. Block TM, Gish R, Guo H, Mehta A, Cuconati A, Thomas London W, et al. Chronic hepatitis B: What should be the goal for new therapies? Antiviral Res. 2013;98:27-34.

4. Liang TJ, Block TM, McMahon BJ, Ghany MG, Urban S, Guo J, et al. Present and future therapies of hepatitis B: From discovery to cure. Hepatology. 2015;62:1893-1908.

5. Bitton Alaluf M, Shlomai A. New therapies for chronic hepatitis B. Liver Int. 2016;36:775-782.

6. Sung JJY, Wong ML, Bowden S, Liew CT, Hui AY, Wong VWS, et al. Intrahepatic hepatitis B virus covalently closed circular DNA can be a predictor of sustained response to therapy. Gastroenterology. 2005;128:1890-1897.

7. Werle-Lapostolle B, Bowden S, Locarnini S, Wursthorn K, Petersen J, Lau G, et al. Persistence of cccDNA during the natural history of chronic hepatitis B and decline during adefovir dipivoxil therapy. Gastroenterology. 2004;126:1750-1758.

8. Dawson MA. The cancer epigenome: Concepts, challenges, and therapeutic opportunities. Science. 2017;355:1147-1152.

9. Bock CT, Schranz P, Schröder CH, Zentgraf H. Hepatitis B virus genome is organized into nucleosomes in the nucleus of the infected cell. Virus Genes. 1994;8:215-229.

10. Bock CT, Schwinn S, Locarnini S, Fyfe J, Manns MP, Trautwein C, et al. Structural organization of the hepatitis B virus minichromosome. J Mol Biol. 2001;307:183-196.

11. Byrd JC. A phase 1 and pharmacodynamic study of depsipeptide (FK228) in chronic lymphocytic leukemia and acute myeloid leukemia. Blood. 2004;105:959-967.

12. Yan H, Zhong G, Xu G, He W, Jing Z, Gao Z, et al. Sodium taurocholate cotransporting polypeptide is a functional receptor for human hepatitis B and D virus. Elife. 2012;1:e00049.

13. Huang HC, Chen CC, Chang WC, Tao MH, Huang C. Entry of hepatitis B virus into immortalized human primary hepatocytes by clathrin-dependent endocytosis. J. Virol. 2012;86:9443-9453.

14. Seeger C, Mason WS. Molecular biology of hepatitis B virus infection. Virology. 2015;479:672-686.

15. Shi L, Li S, Shen F, Li H, Qian S, Lee DHS, et al. Characterization of Nucleosome Positioning in Hepadnaviral Covalently Closed Circular DNA Minichromosomes. J. Virol. 2012;86:10059-10069.

16. Zhu Y, Yamamoto T, Cullen J, Saputelli J, Aldrich CE, Miller DS, et al. Kinetics of hepadnavirus loss from the liver during inhibition of viral DNA synthesis. J. Virol. 2001;75:311-322.

17. Michalopoulos GK, DeFrances MC. Liver regeneration. Science. 1997;276:60-66.

18. Guo JT, Zhou H, Liu C, Aldrich C, Saputelli J, Whitaker T, et al. Apoptosis and regeneration of hepatocytes during recovery from transient hepadnavirus infections. J. Virol. 2000;74:1495-1505.

19. Allweiss L, Volz T, Giersch K, Kah J, Raffa G, Petersen J, et al. Proliferation of primary human hepatocytes and prevention of hepatitis $\mathrm{B}$ virus reinfection efficiently deplete 
nuclear cccDNA in vivo. Gut. 2017;doi:10.1136/gutjnl-2016-312162.

20. Lutgehetmann M, Volz T, Köpke A, Broja T, Tigges E, Lohse AW, et al. In vivo proliferation of hepadnavirus-infected hepatocytes induces loss of covalently closed circular DNA in mice. Hepatology. 2010;52:16-24.

21. Chisari F V, Isogawa M, Wieland SF. Pathogenesis of hepatitis B virus infection. Pathol. Biol. (Paris). 2010;58:258-66.

22. Yuen MF, Wong DKH, Sablon E, Tse E, Ng IOL, Yuan HJ, et al. HBsAg seroclearance in chronic hepatitis B in the Chinese: Virological, histological, and clinical aspects. Hepatology. 2004;39:1694-1701.

23. Loomba R, Liang TJ. Hepatitis B reactivation associated with immune suppressive and biological modifier therapies: current concepts, management strategies and future directions. Gastroenterology. 2017;

24. Schübeler D. Function and information content of DNA methylation. Nature. 2015;517:321-326.

25. Vivekanandan P, Thomas D, Torbenson M. Hepatitis B viral DNA is methylated in liver tissues. J. Viral Hepat. 2008;15:103-107.

26. Zhang Y, Li C, Zhang Y, Zhu H, Kang Y, Liu H, et al. Comparative Analysis of CpG Islands among HBV Genotypes. PLoS One. 2013;8:e56711.

27. Zhang Y, Mao R, Yan R, Cai D, Zhang Y, Zhu H, et al. Transcription of hepatitis B virus covalently closed circular DNA is regulated by $\mathrm{CpG}$ methylation during chronic infection. PLoS One. 2014;9:e110442.

28. Jain S, Chang TT, Chen S, Boldbaatar B, Clemens A, Lin SY, et al. Comprehensive DNA methylation analysis of hepatitis B virus genome in infected liver tissues. Sci. Rep. 2015;5:10478.

29. Vivekanandan P, Daniel HDJ, Kannangai R, Martinez-Murillo F, Torbenson M. Hepatitis $B$ virus replication induces methylation of both host and viral DNA. J. Virol. 2010;84:4321-4329.

30. Arzumanyan A, Reis HM, Feitelson MA. Pathogenic mechanisms in HBV-and HCVassociated hepatocellular carcinoma. Nat. Rev. Cancer. 2013;13:123-135.

31. Barlow DP. Methylation and imprinting: from host defense to gene regulation? Science. 1993;260:309-311.

32. Kouzarides T. Chromatin modifications and their function. Cell. 2007;128:693-705.

33. Allis CD, Berger SL, Cote J, Dent S, Jenuwien T, Kouzarides T, et al. New nomenclature for chromatin-modifying enzymes. Cell. 2007;131:633-636.

34. Rodríguez-Paredes M, Esteller M. Cancer epigenetics reaches mainstream oncology. Nat. Med. 2011;17:330-339.

35. Bedford MT, Richard S. Arginine methylation: an emerging regulatorof protein function. Mol. Cell. 2005;18:263-272.

36. Belloni L, Allweiss L, Guerrieri F, Pediconi N, Volz T, Pollicino T, et al. IFN- $\alpha$ inhibits HBV transcription and replication in cell culture and in humanized mice by targeting the epigenetic regulation of the nuclear cccDNA minichromosome. J. Clin. Invest. 2012;122:529-537.

37. Pollicino T, Belloni L, Raffa G, Pediconi N, Squadrito G, Raimondo G, et al. Hepatitis B virus replication is regulated by the acetylation status of hepatitis B virus cccDNA-bound H3 and H4 histones. Gastroenterology. 2006;130:823-837.

38. Belloni L, Pollicino T, De Nicola F, Guerrieri F, Raffa G, Fanciulli M, et al. Nuclear HBx 
binds the HBV minichromosome and modifies the epigenetic regulation of cccDNA function. Proc. Natl. Acad. Sci. U. S. A. 2009;106:19975-19979.

39. Benhenda S, Ducroux A, Rivière L, Sobhian B, Ward MD, Dion S, et al. Methyltransferase PRMT1 is a binding partner of HBx and a negative regulator of hepatitis B virus transcription. J. Virol. 2013;87:4360-4371.

40. Zhang W, Chen J, Wu M, Zhang X, Zhang M, Yue L, et al. PRMT5 restricts hepatitis B virus replication via epigenetic repression of cccDNA transcription and interference with pgRNA encapsidation. Hepatology. 2017; doi:10.1002/hep.29133.

41. Liu F, Campagna M, Qi Y, Zhao X, Guo F, Xu C, et al. Alpha-interferon suppresses hepadnavirus transcription by altering epigenetic modification of cccDNA minichromosomes. PLoS Pathog. 2013;9:e1003613.

42. Tropberger P, Mercier A, Robinson M, Zhong W, Ganem DE, Holdorf M. Mapping of histone modifications in episomal HBV cccDNA uncovers an unusual chromatin organization amenable to epigenetic manipulation. Proc. Natl. Acad. Sci. U. S. A. 2015;112:E5715-E5724.

43. Ananthanarayanan M, Li Y, Surapureddi S, Balasubramaniyan N, Ahn J, Goldstein J a, et al. Histone H3K4 trimethylation by MLL3 as part of ASCOM complex is critical for NR activation of bile acid transporter genes and is downregulated in cholestasis. Am. J. Physiol. Gastrointest. Liver Physiol. 2011;300:771-781.

44. Allis CD, Jenuwein T. The molecular hallmarks of epigenetic control. Nat. Rev. Genet. 2016;17:487-500.

45. Tropberger P, Pott S, Keller C, Kamieniarz-Gdula K, Caron M, Richter F, et al. Regulation of transcription through acetylation of H3K122 on the lateral surface of the histone octamer. Cell. 2013;152:859-872.

46. Pradeepa MM, Grimes GR, Kumar Y, Olley G, Taylor GCA, Schneider R, et al. Histone $\mathrm{H} 3$ globular domain acetylation identifies a new class of enhancers. Nat. Genet. 2016;48:681-686.

47. Cougot D, Wu Y, Cairo S, Caramel J, Renard C-A, Lévy L, et al. The hepatitis B virus X protein functionally interacts with CREB-binding protein/p300 in the regulation of CREBmediated transcription. J. Biol. Chem. 2007;282:4277-4287.

48. Rivière L, Gerossier L, Ducroux A, Dion S, Deng Q, Michel ML, et al. HBx relieves chromatin-mediated transcriptional repression of hepatitis B viral cccDNA involving SETDB1 histone methyltransferase. J. Hepatol. 2015;63:1093-1102.

49. Decorsière A, Mueller H, van Breugel PC, Abdul F, Gerossier L, Beran RK, et al. Hepatitis B virus $\mathrm{X}$ protein identifies the Smc5/6 complex as a host restriction factor. Nature. 2016;531:386-389.

50. Murphy CM, Xu Y, Li F, Nio K, Reszka-Blanco N, Li X, et al. Hepatitis B virus X protein promotes degradation of SMC5/6 to enhance HBV replication. Cell Rep. 2016;16:2846-2854.

51. Mitra B, Guo H. Hepatitis B virus X protein crosses out Smc5/6 complex to maintain covalently closed circular DNA transcription. Hepatology. 2016;64:2246-2249.

52. Deeks SG. HIV: Shock and kill. Nature. 2012;487:439-440.

53. Ghosh SK, Perrine SP, Williams RM, Faller D V. Histone deacetylase inhibitors are potent inducers of gene expression in latent EBV and sensitize lymphoma cells to nucleoside antiviral agents. Blood. 2012;119:1008-1017.

54. Shim YH, Yoon GS, Choi HJ, Chung YH, Yu E. p16 Hypermethylation in the early stage 
of hepatitis B virus-associated hepatocarcinogenesis. Cancer Lett. 2003;190:213-219.

55. Krawczyk J, Keane N, Freeman CL, Swords R, O’Dwyer M, Giles FJ. 5-Azacytidine for the treatment of myelodysplastic syndromes. Expert Opin. Pharmacother. 2013;14:12551268.

56. Ahuja N, Sharma AR, Baylin SB. Epigenetic Therapeutics: A New Weapon in the War Against Cancer. Annu. Rev. Med. 2016;67:73-89.

57. Henning SM, Wang P, Carpenter CL, Heber D. Epigenetic effects of green tea polyphenols in cancer. Epigenomics. 2013;5:729-741.

58. Huang HC, Tao MH, Hung TM, Chen JC, Lin ZJ, Huang C. (-)-Epigallocatechin-3gallate inhibits entry of hepatitis B virus into hepatocytes. Antiviral Res. 2014;111:100111.

59. Zhong L, Hu J, Shu W, Gao B, Xiong S. Epigallocatechin-3-gallate opposes HBVinduced incomplete autophagy by enhancing lysosomal acidification, which is unfavorable for HBV replication. Cell Death Dis. 2015;6:e1770.

60. Zheng D-L, Zhang L, Cheng N, Xu X, Deng Q, Teng X-M, et al. Epigenetic modification induced by hepatitis $\mathrm{B}$ virus $\mathrm{X}$ protein via interaction with de novo DNA methyltransferase DNMT3A. J. Hepatol. 2009;50:377-387.

61. Chiappinelli KB, Zahnow CA, Ahuja N, Bylin SB. Combining epigenetic and immunotherapy to combat cancer. Cancer Res. 2016;76:1683-1689.

62. Cai D, Wang X, Yan R, Mao R, Liu Y, Ji C, et al. Establishment of an inducible HBV stable cell line that expresses cccDNA-dependent epitope-tagged HBeAg for screening of cccDNA modulators. Antiviral Res. 2016;132:26-37.

63. Falkenberg KJ, Johnstone RW. Histone deacetylases and their inhibitors in cancer, neurological diseases and immune disorders. Nat. Rev. Drug Discov. 2014;13:673-691.

64. Schoggins JW, Rice CM. Interferon-stimulated genes and their antiviral effector functions. Curr. Opin. Virol. 2011;1:519-525.

65. Janssen HLA, Van Zonneveld M, Senturk H, Zeuzem S, Akarca US, Cakaloglu Y, et al. Pegylated interferon alfa-2b alone or in combination with lamivudine for HBeAg-positive chronic hepatitis B: A randomised trial. Lancet. 2005;365:123-129.

66. Kumar A, Darcis G, Van Lint C, Herbein G. Epigenetic control of HIV-1 post integration latency: implications for therapy. Clin. Epigenetics. 2015;7:103.

67. Lucifora J, Xia Y, Reisinger F, Zhang K, Stadler D, Cheng X, et al. Specific and nonhepatotoxic degradation of nuclear hepatitis B virus cccDNA. Science. 2014;343:1221-1228.

68. Noguchi C, Ishino H, Tsuge M, Fujimoto Y, Imamura M, Takahashi S, et al. G to A hypermutation of hepatitis B virus. Hepatology. 2005;41:626-633.

69. Wachowska M, Muchowicz A, Golab J. Targeting Epigenetic Processes in Photodynamic Therapy-Induced Anticancer Immunity. Front. Oncol. 2015;5:176.

70. Youngblood B, Hale JS, Ahmed R. T-cell memory differentiation: Insights from transcriptional signatures and epigenetics. Immunology. 2013;139:277-284.

71. Li H, Chiappinelli KB, Guzzetta A, Easwaran H, Yen RWC, Vatapalli R, et al. Immune regulation by low doses of the DNA methyltransferase inhibitor 5-azacitidine in common human epithelial cancers. Oncotarget. 2014;5:587-598.

72. Buglio D, Georgakis G V., Hanabuchi S, Arima K, Khaskhely NM, Liu YJ, et al. Vorinostat inhibits STAT6-mediated TH2 cytokine and TARC production and induces cell death in Hodgkin lymphoma cell lines. Blood. 2008;112:1424-1433. 
73. Buglio D, Khaskhely NM, Voo KS, Martinez-Valdez H, Liu YJ, Younes A. HDAC11 plays an essential role in regulating OX40 ligand expression in Hodgkin lymphoma. Blood. 2011;117:2910-2917.

74. Xia Y, Stadler D, Lucifora J, Reisinger F, Webb D, Hösel M, et al. Interferon- $\gamma$ and Tumor Necrosis Factor- $\alpha$ Produced by T Cells Reduce the HBV Persistence Form, cccDNA, Without Cytolysis. Gastroenterology. 2016;150:194-205.
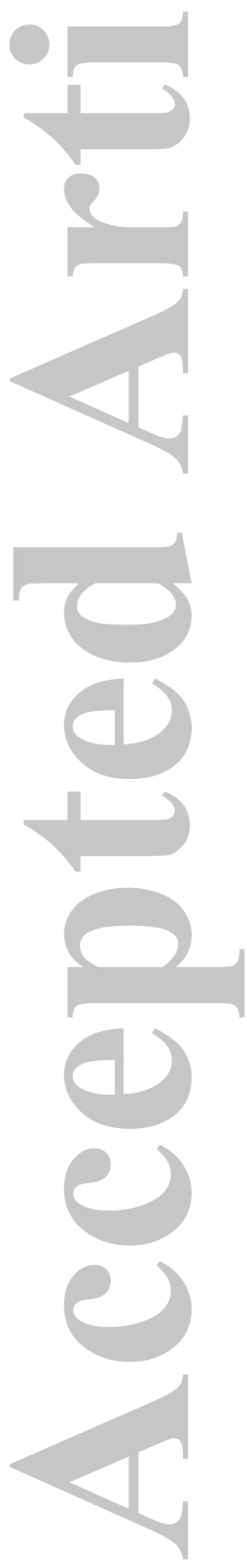


\section{Figure Legend}

Fig 1. HBV life cycle. The major steps in HBV life cycle including entry, trafficking, cccDNA formation, mRNA transcription, pgRNA encapsidation, DNA replication, viral particle assembly and secretion are shown. (Abbreviations: HSPG, heparan sulfate proteoglycan; MVB, multivesicular body; NTCP, sodium taurocholate cotransporting polypeptide.)

Fig 2. Schematic representation of the open reading frames (ORFs) of preC/Core, pol, preS/S, and $\mathrm{X}$ in HBV genome (genotype A, GenBank: AB116086); the four promoters, Cp, Sp1, Sp2, and Xp; the regulatory elements, enhancer 1 and 2 (EN1 and EN2); and three predicted CpG islands.

Fig 3. Schematic representation of modification status of $\mathrm{HBV}$ cccDNA-assoicated histones and the recruitment of chromatin modifying enzymes on cccDNA in relation to $\mathrm{HBx}$. (A) In the presence of HBx, cccDNA-bound histones are hyperacetylated, viral minichromosome is in an open configuration and viral gene transcription is activated, leading to a high level of virus replication. (B) In the absence of $\mathrm{HBx}$, host restriction factors, such as Smc5/6 complex and PRMT1, are loaded on cccDNA. The cccDNA-bound histones are hypoacetylated, viral minichromosome is in a closed configuration and viral gene is transcriptionally repressed, resulting in a low level of virus replication. (Abbreviations: H3/H4 Ac, aceylated histone 3 and 4; CBP, CREB-binding protein; PCAF, p300/CBP-associated factor; PRMT1, protein arginine methyltransferase 1 PRMT5, protein arginine methyltransferase 5; SIRT1, sirtuin 1; HDAC1, histone deaceyltransferase 1; EZH2, enhancer of zeste homolog 2; SUV39H, suppressor of variegation 3-9 Homolog; HP1: heterochromatin protein 1 factors; SETDB1: SET Domain Bifurcated 1. Smc5/6: Structural maintenance of chromosomes protein 5/6.) 


\section{Acknowledgements}

We thank Dr. Xiaobing Shi (MD Anderson Cancer Center) for his critical reading and thoughtful comments on the manuscript. We apologize to numerous investigators whose original contributions could not be cited owing to space limitations.
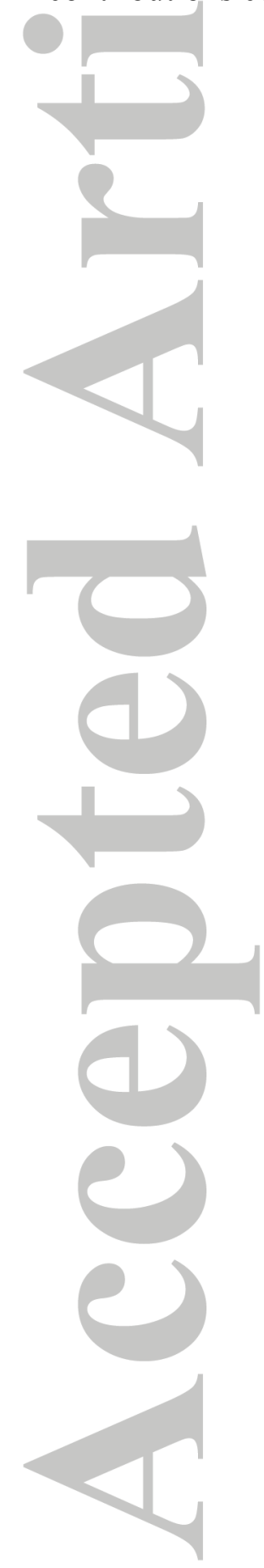
Table 1. List of major epigenetic modifiers in human cells.

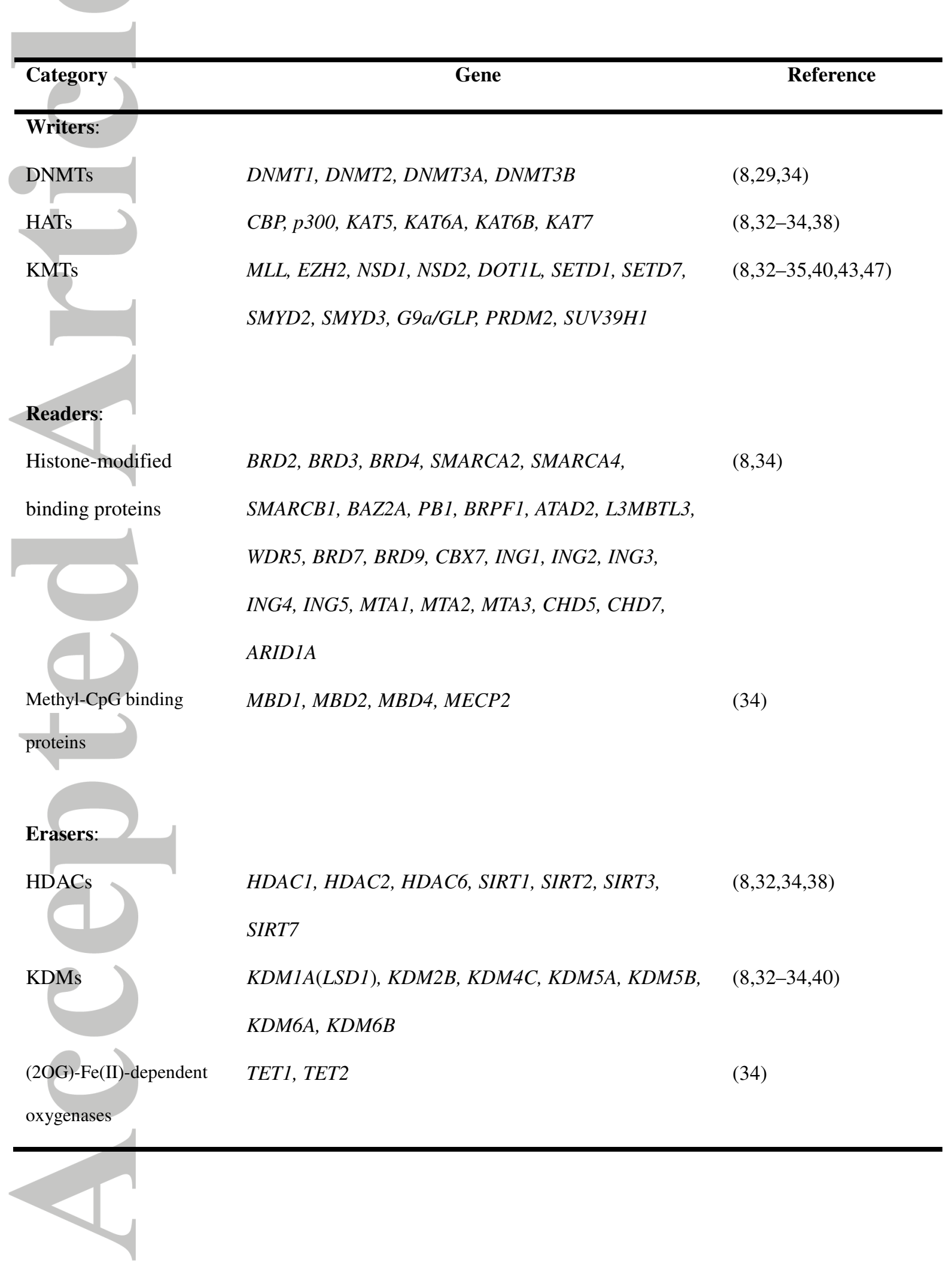

Hepatology

This article is protected by copyright. All rights reserved. 


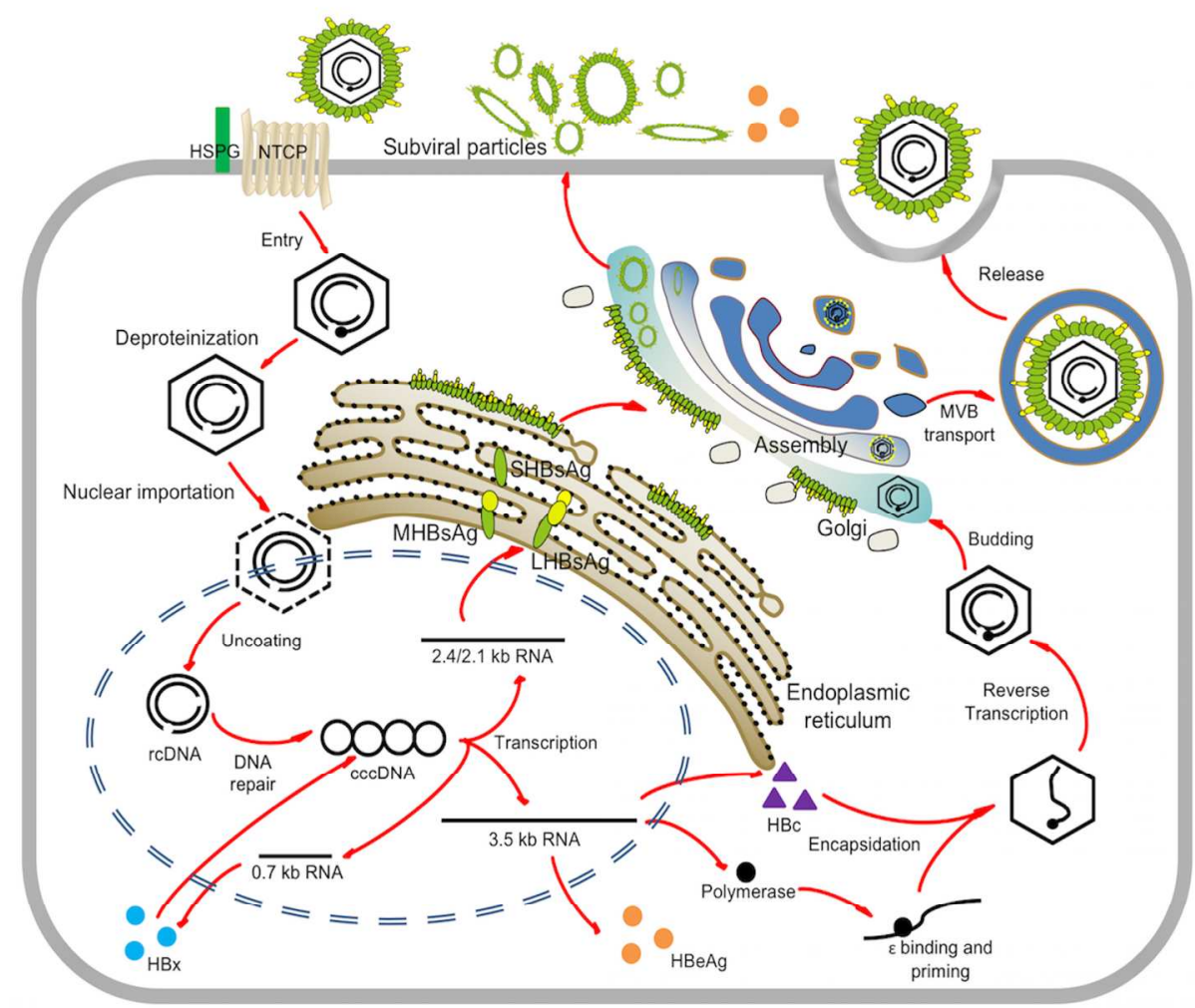

Fig 1. HBV life cycle. The major steps in HBV life cycle including entry, trafficking, cccDNA formation, mRNA transcription, pgRNA encapsidation, DNA replication, viral particle assembly and secretion are shown. (Abbreviations: HSPG, heparan sulfate proteoglycan; MVB, multivesicular body; NTCP, sodium taurocholate cotransporting polypeptide.)

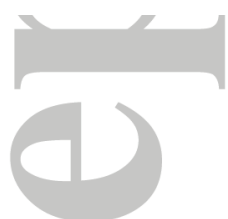

$150 \times 119 \mathrm{~mm}(300 \times 300 \mathrm{DPI})$ 


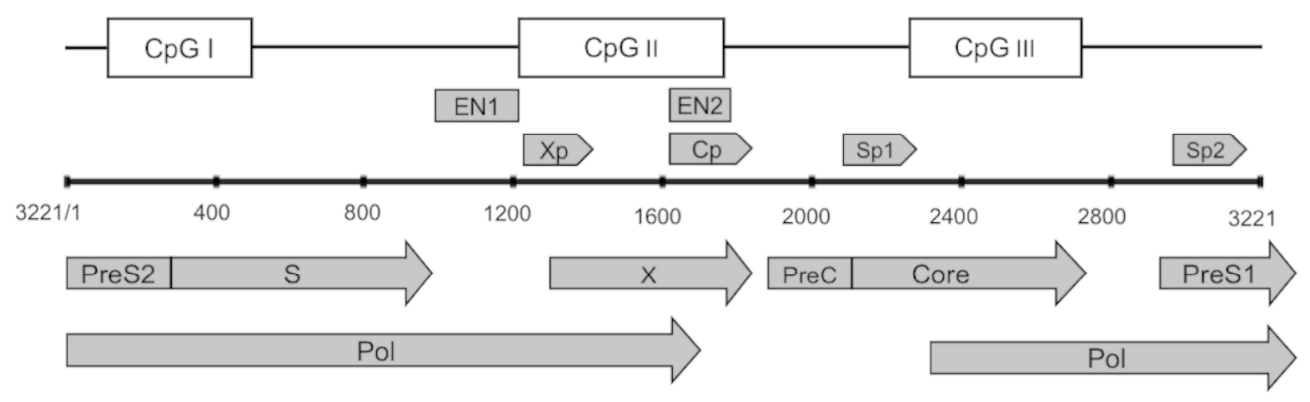

Fig 2. Schematic representation of the open reading frames (ORFs) of preC/Core, pol, preS/S, and $X$ in $\mathrm{HBV}$ genome (genotype A, GenBank: AB116086); the four promoters, $\mathrm{Cp}, \mathrm{Sp} 1, \mathrm{Sp} 2$, and $\mathrm{Xp}$; the regulatory elements, enhancer 1 and 2 (EN1 and EN2); and three predicted CpG islands.

$<$
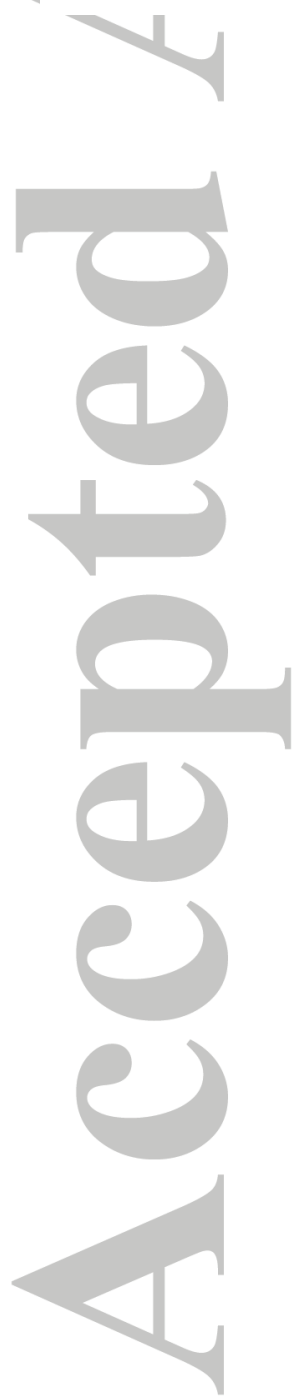

$261 \times 83 \mathrm{~mm}(300 \times 300$ DPI $)$ 


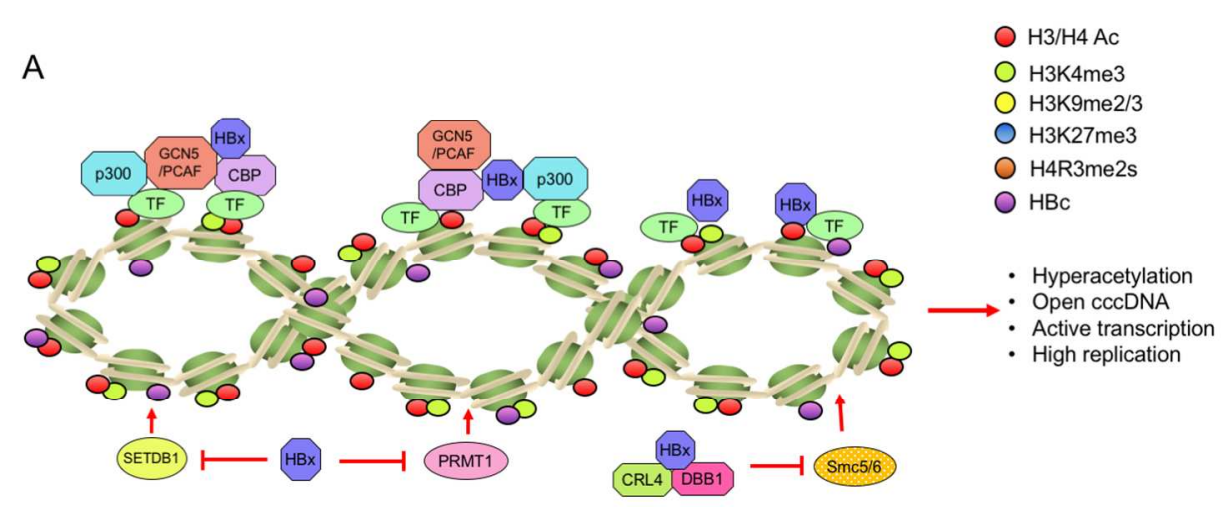

$\mathrm{B}$

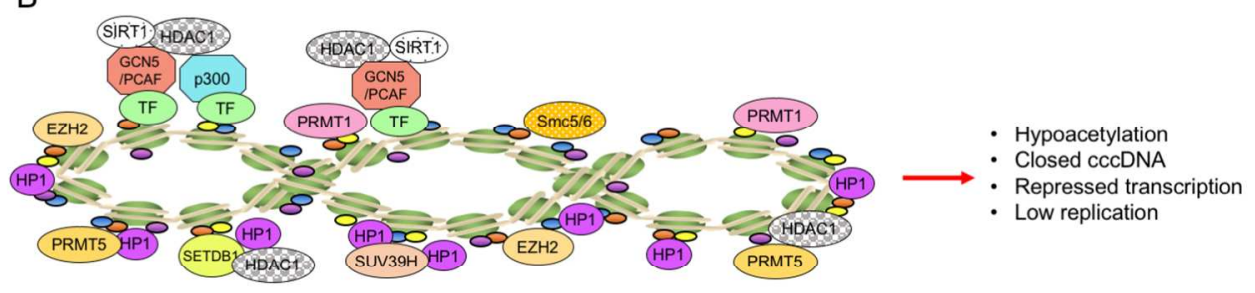

Fig 3. Schematic representation of modification status of HBV ccCDNA-assoicated histones and the recruitment of chromatin modifying enzymes on cccDNA in relation to HBx. (A) In the presence of $\mathrm{HBx}$, cccDNA-bound histones are hyperacetylated, viral minichromosome is in an open configuration and viral - gene transcription is activated, leading to a high level of virus replication. (B) In the absence of HBx, host restriction factors, such as Smc5/6 complex and PRMT1, are loaded on ccCDNA. The ccCDNA-bound histones are hypoacetylated, viral minichromosome is in a closed configuration and viral gene is transcriptionally repressed, resulting in a low level of virus replication. (Abbreviations: $\mathrm{H} 3 / \mathrm{H} 4 \mathrm{Ac}$, aceylated histone 3 and 4; CBP, CREB-binding protein; PCAF, p300/CBP-associated factor; PRMT1, protein arginine methyltransferase 1 PRMT5, protein arginine methyltransferase 5; SIRT1, sirtuin 1; HDAC1, histone deaceyltransferase 1; EZH2, enhancer of zeste homolog 2; SUV39H, suppressor of variegation 3-9 Homolog; HP1: heterochromatin protein 1 factors; SETDB1: SET Domain Bifurcated 1. Smc5/6: Structural maintenance of chromosomes protein $5 / 6$. )

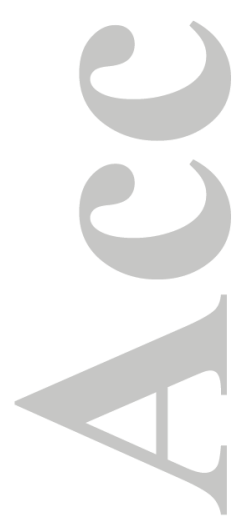

$119 \times 90 \mathrm{~mm}(300 \times 300 \mathrm{DPI})$ 